\title{
CARACTERÍSTICAS CULTURALES ENTRE UNA COOPERATIVA DE AHORRO Y CRÉDITO ECUATORIANA Y EL SECTOR COOPERATIVO NACIONAL
}

\section{CULTURAL CHARACTERISTICS BETWEEN AN ECUADORIAN CREDIT AND SAVINGS COOPERATIVE AND THE NATIONAL COOPERATIVE SECTOR}

\author{
Carrillo Punina Álvaro', Galarza Torres Sandra², Guayasamin Segovia Celio ${ }^{3}$
}

\begin{abstract}
Resumen
Las investigaciones de cultura organizacional adquieren mayor relevancia debido al desarrollo y fundamento de teorías, métodos y estudios empíricos que revelan la influencia que esta tiene en el desempeño. Así, el estudio reciente de Carrillo-Punina confirma la relación entre las tipologias culturales orientadas a los resultados con algunas variables del desempeño financiero en el sector de cooperativas de ahorro y crédito ecuatoriano. De este modo, continuando con el estudio, se desarrolla la investigación de tipología cultural en una cooperativa de ahorro y crédito del mismo segmento que se caracteriza por la amplia cobertura geográfica. Se aplica el cuestionario de Felcman y Góngora a una muestra de 377 funcionarios de la cooperativa que representa el 76\% de la población y fiabilidad Alpha de Cronbach de 0,978. Los resultados indican el predominio de la cultura exigente, seguido de la tipología apática; sin embargo, el sector presenta el dominio cultural exigente e integrativo lo que conduce al planteamiento de una nueva hipótesis de investigación: las cooperativas con fortaleza financiera tipo A presentan tipologias culturales exigentes e integrativas y, aquellas con fortaleza financiera tipo b, se identifican con tipologias exigentes o integrativas y apáticas.
\end{abstract}

\section{Palabras Claves}

Cultura organizacional; clima organizacional; valores centrales

\begin{abstract}
Organizational culture researches acquire greater importance due to the development and Foundation of theories, methods and empirical studies that reveal the influence this has on performance. Thus, the recent study of CarrilloPunina confirms the relationship between cultural kinds-oriented results with some variables from the financial performance in the sector of Ecuadorian credit unions. Thus, continuing with the study develops the research of cultural typology in cooperative credit unions in the same segment that is characterized by the wide geographic coverage. Applies Felcman and Góngora questionnaire to a sample of 377 officers of the cooperative which represents $76 \%$ of the population and reliability 0.978 Cronbach's Alpha. Results indicate the prevalence of demanding culture, followed by the APATHETIC typology, however, the SECTOR presents the cultural domain demanding and INTEGRATIVE, leading to a new hypothesis of research approach: cooperative with financial strength type a present cultural type demanding and INTEGRATIVE and those with type B financial strength, identify types demanding or INTEGRATIVE and APATHETIC.
\end{abstract}

Keywords

Organizational culture; organizational climate; core values

1 Universidad de las Fuerza Armadas ESPE. apcarrillo@espe.edu.ec

$2 \quad$ Universidad de las Fuerzas Armadas ESPE. spgalarza@espe.edu.ec

3 Universidad de las Fuerzas Armadas ESPE. cfguayasamin@espe.edu.ec 


\section{Introducción}

Durante los últimos años, la cultura organizacional se ha convertido en un ámbito de investigación científica y en un factor de gestión para desarrollar estrategias enfocadas al logro de resultados y la supervivencia de la organización a largo plazo (Carrillo, 2016). Para Góngora, Nobile y Reija (2014), la cultura organizacional es el conjunto de normas, creencias, valores, costumbres, rituales, lenguajes, artefactos y presunciones básicas presentes en una organización. Así mismo, autores como Sethia y Von Glinow (1985) señalan que la cultura organizacional es "un modelo compartido y relativamente duradero de valores básicos, creencias y presunciones en una organización" (p. 403).

Gálvez y García (2011) desarrollan un estudio sobre la relación entre cultura organizacional y rendimiento. Para lo cual, analizan 60 MiPymes tecnológicas de Cali mediante la escala de valores de Likert y el modelo de Cameron y Quinn (1999). Los resultados destacan que existe relación positiva y negativa entre el tipo de cultura dominante con el rendimiento de la empresa. Por otro lado, Gómez y Ricardo (2012) utilizan la encuesta de Daniel Denison (1997, 2001), que contiene 60 preguntas estructuradas en la escala de Likert; en esta se evidencia la relación entre componentes de la cultura organizacional con algunas características de la innovación.

Algunos autores como Shein (1988), Cameron y Quinn (2011), Denison (1997), Kotter y Heskett (1992), Sethia y Von Glinow (1985), entre otros, han desarrollado modelos sobre el diagnóstico de tipología cultural dominante en los grupos sociales. Sin embargo, Felcman y Góngora (1995) como cita Marrón (2015), desarrollan un instrumento que define la tipología cultural dominante, de acuerdo con la orientación que tiene la organización hacia las personas y hacia los resultados y, se sustenta en diez dimensiones de la gestión. Los referidos tipos culturales de Felcman y Góngora se definen en paternalista, apático, anómico, exigente e integrativo; y las dimensiones se presentan en dirección, cambio, planeación, remuneración, control, información, horizonte temporal, contenido de la tarea, posibilidades de éxito y criterios de éxito.

Por otro lado, las cooperativas de ahorro y crédito ecuatorianas son un elemento vital de sistema financiero del país ${ }^{4}$; han permanecido en el mercado financiero por más de treinta años y, han superado crisis de toda indole. Además, representan estructuras organizacionales formales, ya que el cumplimiento de metas y normas son una prioridad de la función del negocio. La cooperativa de ahorro y crédito objeto de estudio pertenece al segmento uno que se compone de 26 cooperativas, con montos superiores a 200 millones de dólares en activos, y poseen una gestión de alto desempeño financiero que se demuestra en los resultados de los últimos años (Galarza Torres, 2019).

Carrillo-Punina (2019), en su tesis doctoral titulada Cultura organizacional y desempeño financiero en las cooperativas de ahorro y crédito ecuatorianas, determina que el segmento uno ${ }^{5}$ de estas cooperativas posee culturas dominantes exigentes e integrativas, con una fuerte orientación hacia los resultados, sin descuidar la orientación hacia las personas. El autor identifica una fuerte relación directa entre las tipologías culturales exigentes e integrativas con algunos indicadores del desempeño financiero, a saber, el volumen de créditos, depósitos a

$4 \quad$ El sistema financiero es el conjunto de instituciones, medios y mercados en donde se presentan transacciones financieras, por ende, existen colocadores y captadores de fondos que por medio de las decisiones de consumo y ahorro facilitan la transferencia de recursos tal como se cita en Carrillo-Punina (2019) a Mishkin (2008).

5 Las cooperativas que superan los 80 millones de dólares en activos pertenecen al segmento uno según la clasificación de la Junta de Política y Regulación Monetaria y Financiera del Ecuador, resolución 038-2015-F, 13 de febrero de 2015. 
la vista y a plazo, liquidez y rentabilidad. De este modo, cuanto más fuerte es el dominio de las tipologías exigentes e integrativas, más favorables son los indicadores de desempeño financiero citados; $y$, cuanto más dominan las culturas paternalistas, apáticas y anómicas, más desfavorables son los indicadores financieros alcanzados en estas organizaciones.

En toda su historia, la República del Ecuador se ha caracterizado por vivir en diversas situaciones de crisis que han sido provocadas por el entorno económico, político, ambiental, tecnológico y social. Aspectos que de una u otra forma, han afectado el funcionamiento normal de los grupos sociales (Carrillo-Punina y Galarza-Torres, 2018). Por ende, la cultura organizacional definida como un conjunto de artefactos, valores y presunciones básicas, que moldean el comportamiento de los miembros de la organización y que son compartidas y aceptadas por cada uno de ellos, se convierte en un factor crítico del éxito que permite a la organización afrontar crisis y amenazas, así como también, aprovechar oportunidades (Sethia y Von Glinow, 1985; Shein, 1988; Kotter y Heskett, 1995; Hofstede, 1999; Pfeffer, 2000; García, 2006; Adeyoyin, 2006; Vargas, 2007; Miquilena y Paz, 2008; Rodríguez, 2009; Cameron y Quinn, 2011; Martínez, 2012; Felcman, 2015; Góngora, Pérez y Gelonch, 2017; Carrillo-Punina, 2019; Galarza-Torres, Carrillo-Punina y Carrillo-Punina, 2019).

Por lo expuesto, el presente estudio se desarrolla para identificar la cultura dominante en una Cooperativa de Ahorro y Crédito que se caracteriza por tener una amplia cobertura nacional a través de oficinas, con el propósito de establecer la situación cultural actual y deseada y el índice de frustración. También, se comparan los resultados obtenidos del estudio de la cooperativa y aquellos del sector generados por CarrilloPunina (2019). Es así que la investigación establece, en el primer apartado, la introducción que contiene los problemas y objetivos de estudio; luego se describe la metodología empleada para el levantamiento y tratamiento de datos. A continuación, se revelan los resultados alcanzados $y$, finalmente, se exponen las conclusiones y nuevas líneas de investigación.

\section{Metodología}

La investigación es de tipo cualitativa y cuantitativa, ya que utiliza el instrumento de Felcman y Góngora (1995), descrito por Marrón (2015) que ayuda a diagnosticar la tipología de cultura organizacional presente y deseada. De igual forma, se aplica una entrevista administrada a la dirección de talento humano de la Cooperativa, con el fin de profundizar acerca de algunos resultados obtenidos del tratamiento de la encuesta.

La cooperativa de ahorro y crédito seleccionada pertenece al segmento uno, según clasificación de la Superintendencia de Economía Popular y Solidaria del Ecuador, posee activos mayores a ochenta millones de dólares; se encuentra en el sistema financiero por más de treinta años; la cobertura geográfica comprende cerca de cuarenta oficinas ubicadas en todo el país, y presenta un nivel de calificación de riesgo financiero de $\mathrm{BBB}^{+6}$ (SEPS, 2018). Para el efecto, se levantan y tratan 377 encuestas aplicadas al personal de la cooperativa de todo grado jerárquico que representa una muestra equivalente al $76 \%$ de la población total, con un error muestral de 2,47\%, probabilidad de ocurrencia del $5 \%$, nivel de confianza del 98 $\%$ y fiabilidad Alpha de Cronbach del 0.978. El tratamiento de datos se lo hace en el paquete estadístico SPSS versión 23.

6 BBB+: Se considera que claramente esta institución tiene buen crédito. Aunque son evidentes algunos obstáculos menores, éstos no son serios y/o son perfectamente manejables a corto plazo (SEPS, 2018). 


\section{Resultados}

\section{Tipología cultural actual de la coopera- tiva ahorro y crédito}

El estudio determina que la tipología cultural dominante en la cooperativa de estudio es exigente con el $29 \%$ de percepción del personal que labora en los distintos niveles jerárquicos, seguido de una tipología apática concentrada en el 25,2\% de la organización, como se observa en la tabla 1. Por tanto, la cultura organizacional, en teoría exigente, se caracteriza en la cooperativa porque se hace hincapié en un vínculo laboral calificado como demandante e inflexible, esencialmente basado en la fijación de objetivos y la exigencia de su cumplimiento. De este modo, se incentiva la competencia interna y la insensibilidad frente a las necesidades de los otros. Se prioriza la eficiencia y los aportes individuales, se juzga y se premia o castiga por lo que se produce, y se coloca un especial énfasis en los resultados. Los sistemas de premios y castigos se establecen sobre la base del cumplimiento de objetivos fijados, poniendo acento en una fuerte remuneración variable que prevea el grado de realización de los mismos.

Precisamente, la segunda tipología dominante es apática que teóricamente se expresa como sinónimo de desinterés y falta de involucramiento, la indiferencia por el comportamiento de la gente, el estado de incertidumbre y confusión, la pérdida de entusiasmo, la ausencia de recompensas para premiar los éxitos, la poca seguridad laboral que a su vez determina el escaso interés por el futuro y la preocupación excesiva por lo inmediato centrada principalmente en evitar el despido. Se tiene la sensación de estar a la deriva.

Tabla 1. Tipo de cultura organizacional presente

\begin{tabular}{|l|c|}
\hline \multicolumn{1}{|c|}{ Tipología cultural } & Porcentaje \\
\hline Paternalista & $17,0 \%$ \\
\hline Apática & $\mathbf{2 5 , 2 \%}$ \\
\hline Anómica & $12,5 \%$ \\
\hline Exigente & $29,0 \%$ \\
\hline Integrativa & $16,3 \%$ \\
\hline Total & $100,0 \%$ \\
\hline
\end{tabular}

Fuente: Elaboración de los autores

En respecto con las dimensiones dominantes que se identifican en la tipología cultural de la cooperativa base de este estudio, se establecen los resultados en la tabla 2. Por tanto, las dimensiones particulares de la cooperativa se definen de a siguiente manera:

1) Cambio: esta dimensión presenta el 39,18\% de dominio en la organización con características paternalistas; es decir, "se observan las cosas que cambian en la realidad y se adapta a los cambios en forma gradual";

2) Dirección: el dominio en la relación entre el jefe y sus subordinados en la organización de análisis se presenta en un 26,48\% con rasgos integrativos; es decir, "cuando se debe tomar una decisión importante el jefe reúne a sus colaboradores para discutir el tema. Considera todos los puntos de vista y trata de que todos estén de acuerdo con la decisión. Si todos, incluido él mismo, están 
de acuerdo, toma esa decisión como propia. Si no hay consenso, toma la decisión que considera más adecuada".

3) Remuneración: la forma como se recompensa al personal por los logros alcanzados en la cooperativa tiene dominio en el $29,19 \%$ con características integrativas; por eso, "además de la remuneración fija existe otra variable según los resultados individuales y grupales obtenidos".

4) Planeación: el sistema de planificación de la organización es exigente, con el 29,63\% de la percepción de sus miembros; por esto, en la cooperativa "se fijan los objetivos y se evalúa su cumplimiento a través de resultados".

5) Información: el flujo de información entre los miembros de la cooperativa presenta rasgos apáticos en el 39,13\%; entonces, "se encuentra disponible solo la información que establecen las normas y procedimientos".

6) Control: las maneras de aceptar los errores tienen características exigentes; por ello, "se llama la atención y se establece distancia entre los objetivos comprometidos y su cumplimiento".
7) Horizonte temporal: en este caso se presenta el dominio compartido entre rasgos exigentes e integrativos, es así que la organización centra sus acciones orientadas hacia el corto y largo plazos.

8) Contenido de la tarea: la estructura de las tareas y procesos dentro de la cooperativa se caracteriza por la presencia dominante de rasgos exigentes, 38,84\%; es decir, "las tareas son fuertemente desafiantes y competitivas, por tanto, persiguen alcanzar los objetivos establecidos".

9) Posibilidades de éxito: Esta dimensión revela un claro dominio de rasgos apáticos en el 34,93\%, precisamente, la cooperativa se centra en mantener fortalezas en "solidez económica, prudencia en las decisiones $y$, confiabilidad en los procesos administrativos"

10) Criterios de éxito: el $38,84 \%$ de los miembros de la cooperativa señalan que en esta dimensión predominan rasgos exigentes, así, se tienen como factores críticos del éxito "los objetivos están claros y la gente busca obtener resultados concretos en función de dichos objetivos".

Tabla 2. Dimensiones dominantes de cultura organizacional

\begin{tabular}{|c|c|c|c|c|c|}
\hline & Paternalista & Apática & Anómica & Exigente & Integrativa \\
\hline Cambio & $39,18 \%$ & $21,35 \%$ & $8,19 \%$ & $19,01 \%$ & $12,28 \%$ \\
\hline Dirección & $14,65 \%$ & $22,82 \%$ & $14,08 \%$ & $21,97 \%$ & $26,48 \%$ \\
\hline Remuneración & $7,23 \%$ & $28,03 \%$ & $13,29 \%$ & $22,25 \%$ & $29,19 \%$ \\
\hline Planeación & $22,79 \%$ & $25,64 \%$ & $11,40 \%$ & $29,63 \%$ & $10,54 \%$ \\
\hline Información & $16,23 \%$ & $39,13 \%$ & $4,35 \%$ & $18,55 \%$ & $21,74 \%$ \\
\hline Control & $8,33 \%$ & $14,66 \%$ & $17,24 \%$ & $47,99 \%$ & $11,78 \%$ \\
\hline Horizonte temporal & $7,46 \%$ & $24,18 \%$ & $10,15 \%$ & $29,25 \%$ & $28,96 \%$ \\
\hline Contenido de la tarea & $17,10 \%$ & $20,58 \%$ & $17,39 \%$ & $38,84 \%$ & $6,09 \%$ \\
\hline Posibilidades de éxito & $20,00 \%$ & $34,93 \%$ & $11,34 \%$ & $23,58 \%$ & $10,15 \%$ \\
\hline Criterios de éxito & $17,10 \%$ & $20,58 \%$ & $17,39 \%$ & $38,84 \%$ & $6,09 \%$ \\
\hline
\end{tabular}

Fuente: Elaboración de los autores

Precisamente, el estudio de CarrilloPunina (2019) revela que el sector cooperativo segmento uno presenta una tipología cultural exigente con el $43,49 \%$ de percepción. Por con- 
siguiente, dominan las dimensiones con rasgos exigentes de dirección, cambio, remuneración, información, control, horizonte temporal y posibilidades de éxito, y con rasgos integrativos las dimensiones de planeación, contenido de la tarea y criterios de éxito. En consecuencia, la cultura organizacional en el SECTOR está orientada a los resultados y a las personas, es así que el 80,08\% del sector posee un alta orientación hacia los resultados. Es importante destacar que, en las cooperativas de ese estudio, seleccionadas por muestreo intencionado, se identifican calificaciones de riesgo financiero de tipo $A^{7}$, es decir, con altas fortalezas financieras.

De acuerdo con estas tipologías dominantes en el sector y en la cooperativa de este estudio, se formula una nueva hipótesis "las coo- perativas con fortaleza financiera Tipo A presentan tipologías culturales exigentes e integrativas $y$, aquellas con fortaleza financiera b se identifican con tipologías exigentes o integrativas y apáticas".

\section{Tipología cultural deseada de la coope- rativa ahorro y crédito}

Las percepciones de los miembros de la Cooperativa sobre la cultura organizacional esperada se orientan hacia una tipología integrativa en el $31,7 \%$, y se busca el predominio de la cultura exigente en el 29,7\%. Por tanto, se persigue una mayor orientación hacia las personas, sin dejar de lado la orientación hacia los resultados que ya se manifiestan en la tipología cultural presente.

Tabla 3. Tipo de cultura organizacional deseada

\begin{tabular}{|l|c|}
\hline \multicolumn{1}{|c|}{ Tipología cultural } & Porcentaje \\
\hline Paternalista & $11,8 \%$ \\
\hline Apática & $20,7 \%$ \\
\hline Anómica & $6,1 \%$ \\
\hline Exigente & $29,7 \%$ \\
\hline Integrativa & $31,7 \%$ \\
\hline Total & $100,0 \%$ \\
\hline
\end{tabular}

Fuente: Elaboración de los autores

La cultura organizacional deseada se caracteriza por la búsqueda de dimensiones culturales con rasgos dominantes integrativos en el cambio, remuneración, planeación, información, control y horizonte temporal; mientras que con rasgos exigentes se anhelan las dimensiones de dirección y contenido de la tarea. Sin embargo, permanece el deseo de posibilidades de éxito con rasgos apáticos, tal como se describe en la entrevista, esto se debe a la búsqueda constante de solidez económica, prudencia en las decisiones, y confiabilidad en los procesos administrativos, como únicas metas definidas para la organización.

$7 \quad$ AAA: La situación de la institución financiera es muy fuerte y tiene una sobresaliente trayectoria de rentabilidad, lo cual se refleja en una excelente reputación en el medio, muy buen acceso a sus mercados naturales de dinero y claras perspectivas de estabilidad. Si existe debilidad o vulnerabilidad en algún aspecto de las actividades de la institución, esta se mitiga enteramente con las fortalezas de la organización (SEPS, 2018). 
Tabla 4. Dimensiones culturales deseadas

\begin{tabular}{|l|l|l|l|l|l|}
\hline & \multicolumn{1}{|c|}{ Paternalista } & \multicolumn{1}{|c|}{ Apática } & \multicolumn{1}{c|}{ Anómica } & \multicolumn{1}{c|}{ Exigente } & \multicolumn{1}{|c|}{ Integrativa } \\
\hline Cambio & 20,60 & 15,11 & 1,37 & 13,19 & $\mathbf{4 9 , 7 3}$ \\
\hline Dirección & 24,10 & 8,86 & 6,37 & $\mathbf{4 9 , 3 1}$ & 11,36 \\
\hline Remuneración & 13,61 & 30,00 & 4,72 & 19,17 & $\mathbf{3 2 , 5 0}$ \\
\hline Planeación & 3,85 & 37,91 & 2,20 & $\mathbf{3 9 , 5 6}$ & 16,48 \\
\hline Información & 4,99 & 3,32 & 22,16 & 13,02 & $\mathbf{5 6 , 5 1}$ \\
\hline Control & 7,20 & 30,75 & 3,32 & 26,59 & $\mathbf{3 2 , 1 3}$ \\
\hline Horizonte temporal & 0,84 & 24,30 & 3,63 & 29,89 & $\mathbf{4 1 , 3 4}$ \\
\hline Contenido de la tarea & 8,50 & 5,67 & 12,46 & 46,46 & 26,91 \\
\hline Posibilidades de éxito & 14,44 & 42,22 & 2,78 & 18,89 & 21,67 \\
\hline Criterios de éxito & 19,89 & 8,81 & 1,99 & 41,19 & 28,13 \\
\hline
\end{tabular}

Fuente: Elaboración de los autores

De igual manera, la cultura organizacional esperada en el sector es exigente [43,45\%] e, integrativa [42,68\%]. Al mismo tiempo, las dimensiones dominantes se concentran en mayor cantidad dentro de los rasgos integrativos $y$, las dimensiones de cambio, horizonte temporal y posibilidades de éxito presentan características exigentes. La orientación hacia los resultados que se desea alcanzar es del 86,13\% (CarrilloPunina, 2019).

\section{Indice de frustración cultural}

Según Felcman y Blutman (2011), el concepto de índice de frustración cultural colectiva es la distancia entre una situación cultural existente y una situación cultural requerida. En consecuencia, se determina que la brecha cultural organizacional, en la cooperativa, está dada por un índice de crecimiento positivo hacia la tipología integrativa en las dimensiones de cambio, información, control, horizonte temporal, contenido de la tarea, posibilidades de éxito y criterios de éxito. En tanto, se espera un crecimiento de rasgos exigentes en dirección, reducción de características exigentes en la dimensión de control, y el decrecimiento de rasgos paternalistas en planeación y apáticos en el sistema de información.

Por la parte, se espera un dominio cultural integrativo que pasa del $16.3 \%$ al $31.7 \%$, entonces, existe una amplia brecha entre lo actual y deseado que representa el 15.4\%; empero, en el estudio del sector la brecha apenas es del 6.09\%. Por consiguiente, esta cooperativa, debido al dominio cultural exigente y apático, se encuentra en un horizonte de más largo tiempo para llegar a una tipología integrativa. Sin embargo, en la cooperativa, el dominio cultural exigente deseado y real está dado por el $29.7 \%$ y $29.0 \%$, respectivamente; es decir, generan una brecha mínima del $0.7 \%$, así, la organización mantiene el porcentaje dominante de rasgos exigentes.

Tabla 5. Índice de frustración (\%)

\begin{tabular}{|l|l|l|l|l|l|}
\hline & \multicolumn{1}{|c|}{ Paternalista } & \multicolumn{1}{c|}{ Apática } & \multicolumn{1}{c|}{ Anómica } & Exigente & \multicolumn{1}{c|}{ Integrativa } \\
\hline Cambio & $-18,58$ & $-6,24$ & $-6,81$ & $-5,82$ & $\mathbf{3 7 , 4 4}$ \\
\hline Dirección & 9,45 & $-13,95$ & $-7,71$ & $\mathbf{2 7}, 34$ & $-15,12$ \\
\hline Remuneración & 6,39 & 1,97 & $-8,57$ & $-3,09$ & 3,31 \\
\hline
\end{tabular}




\begin{tabular}{|l|l|l|l|l|l|}
\hline Planeación & $-\mathbf{1 8 , 9 5}$ & 12,27 & $-9,20$ & 9,93 & 5,94 \\
\hline Información & $-11,25$ & $\mathbf{- 3 5 , 8 1}$ & 17,81 & $-5,53$ & $\mathbf{3 4 , 7 7}$ \\
\hline Control & $-1,13$ & 16,09 & $-13,92$ & $\mathbf{- 2 1 , 4 0}$ & $\mathbf{2 0 , 3 5}$ \\
\hline Horizonte temporal & $-6,62$ & 0,12 & $-6,52$ & 0,63 & $\mathbf{1 2 , 3 9}$ \\
\hline Contenido de la tarea & $-8,60$ & $-14,91$ & $-4,93$ & 7,62 & $\mathbf{2 0 , 8 3}$ \\
\hline Posibilidades de éxito & $-5,56$ & 7,30 & $-8,57$ & $-4,69$ & $\mathbf{1 1 , 5 2}$ \\
\hline Criterios de éxito & 2,78 & $-11,77$ & $-15,40$ & 2,35 & $\mathbf{2 2 , 0 4}$ \\
\hline
\end{tabular}

Fuente: Elaboración de los autores

\section{Conclusiones}

La cooperativa de ahorro y crédito que pertenece al segmento uno, con activos superiores a los ochenta millones de dólares, presenta una tipología cultural compuesta por el dominio exigente y apático. En consecuencia, tiene orientación hacia los resultados; sin embargo, existe una subcultura con rasgos apáticos dentro de la organización. Por su lado, en el sector se destaca el predominio de la tipología cultural exigente e integrativa y carencia de grupos dominantes apáticos.

Las dimensiones culturales dominantes en la cooperativa presentan rasgos integrativos en dirección, remuneración y horizonte temporal; mientras que con rasgos exigentes se tienen planeación, control, horizonte temporal, contenido de la tarea y criterios de éxito. Por su parte, predominan rasgos apáticos en las dimensiones de información y posibilidades de éxito y características paternalistas en la dimensión de cambio. Este factor puede ser el efecto de una alta rotación de personal y una causa del desempeño financiero con calificación $B$.

La cultura organizacional deseada por los miembros de la cooperativa se centra en la orientación hacia los resultados y orientación hacia las personas; es decir, se anhela una cultura de tipo integrativa. Por ende, se esperan fortalecer los rasgos dominantes integrativos de las dimensiones de cambio, remuneración, planeación, información, con- trol y horizonte temporal. Asimismo, el incremento de rasgos exigentes en dirección y contenido de la tarea. También se espera el crecimiento de rasgos apáticos en la dimensión de posibilidades de éxito; este último aspecto se debe a la fijación de metas firmes en solidez económica, prudencia en las decisiones y confiabilidad en los procesos administrativos.

En esta investigación se aporta teórica y empíricamente con la aplicación del modelo planteado por Felcman y Góngora, por consiguiente, la gestión debe hacer hincapié en el trabajo sobre las dimensiones culturales que conducen hacia diversas maneras de proceder, trabajar y lograr resultados, ya que la cultura organizacional y sus manifestaciones aportan en la toma de decisiones. Por último, se plantea la hipótesis "las cooperativas con fortaleza financiera tipo A presentan tipologías culturales exigentes e integrativas, y aquellas con fortaleza financiera tipo B se identifican con tipologías culturales exigentes o integrativas y apáticas".

\section{Referencias bibliográficas}

Adeyoyin, S. (2006). Managing the Library's Corporate Culture for Organizational Efficiency, Productivity, and Enhanced Service. Library Philosophy and Practice, 8 (2), 1-14.

Cameron, K. Y Quinn, R. (2011). Diagnosing and changing organizational culture. Based on the competing value framework. United States of America: Josey Bass. 
Carrillo Punina, A. (2016). Medición de la Cultura Organizacional. Ciencias Administrativas, (8). Recuperado a partir de https://revistas.unlp.edu.ar/CADM/article/view/2637

Carrillo-Punina, Á. y Galarza-Torres, S. (2018). Tipología de cultura organizacional en una cooperativa de ahorro y crédito ecuatoriana. COODES, 6(1), p. 81-97. Obtenido de http://coodes.upr.edu.cu/index.php/coodes/article/view/170

Carrillo-Punina, Á. (13 de marzo de 2019). Cultura organizacional y desempeño financiero en las cooperativas de ahorro y crédito ecuatorianas. Tesis doctoral. La Plata: Universidad Nacional de La Plata. Facultad de Ciencias Económicas. Argentina. http:// sedici.unlp.edu.ar/handle/10915/75026.

Denison, D. (1997). Corporate culture and organizational effectiveness. United States of America: Library of Congress Catalogingin-Publication Data.

Felcman, I., y Blutman, G. (2011). Nuevos modelos de gestión pública: Cultura, liderazgo y tecnologias de gestiòn después del "big bang" paradigmático. Buenos Aires: Temas Grupo Editorial SRL. doi:http://www.saludcolectiva-unr.com.ar/docs/SC-244.pdf

Galarza-Torres, S.; Carrillo-Punina, Á. y CarrilloPunina, L. (2019). Estilos de liderazgo en las cooperativas de ahorro y crédito ecuatorianas: modelo lineal de equilibrio en resultados. ECA SINERGIA, 10(2). Recuperado a partir de https://revistas.utm. edu.ec/index.php/ECASinergia/article/ view/1659/1993.

Galarza-Torres, S. (21 de agosto 2019) Liderazgo y desempeño en las cooperativas de ahorro y crédito ecuatorianas Un acercamiento desde las contribuciones teóricas clásicas y contemporáneas Tesis doctoral. La Plata: Universidad Nacional de La Plata. Facultad de Ciencias Económicas. Argentina. http://sedici.unlp.edu.ar/handle/10915/79854

Gálvez, J. y García, D. (2011). Cultura, Innovación, Intraemprendimiento y Rendimiento en las MIPYME de Colombia. Tesis Doctoral. Cartagena, Colombia: Universidad Politécnica de Cartagena
García, C. (2006). Una aproximación al concepto de cultura organizacional. Universitas Psychologica, 5 (1), 163-174.

Góngora, N., Pérez-Ruffa, A. y Gelonch, G. (2017). El jefe inmediato. CONLAD 2017 (págs. 1-11). Misiones: CONLAD.

Góngora, Norberto H.; Nóbile, C.; Reija S. Estudio comparativo de la cultura organizacional. Ciencias Administrativas, núm. 4, julio-diciembre, 2014, pp. 51-65 Universidad Nacional de La Plata Buenos Aires, Argentina

Gómez, I. y Ricardo, R. (2012). Cultura organizacional: organización sectorial en Bogotá. Revista Facultad de Ciencias Económicas: Investigación y Reflexión, 20 (2), 19-41.

Hofstede, G. (1999). Culturas y Organizaciones. El software mental. La cooperación internacional y su importancia para la supervivencia. Madrid: Alianza Editorial.

Kotter, J. y Heskett, J. (1995). Cultura de empresa y rentabilidad. Madrid: Ediciones Díaz de Santos S.A.

Sethia, N. y Von Glinow, M. A. (1985). Arriving at Four Cultures by Managing the Reward System. En: Kilmann y otros: Gaining Control of the Corporate Culture. Jossey Bass, San Francisco, EE.UU.

Schein, E. (1988). La cultura empresarial y el liderazgo una visión dinámica. Barcelona España: Plaza \& Janés.

SEPS. (2018, julio 23). Superintendencia de Economía Popular y solidara. Retrieved from Calificacion de Riesgos: http://www. seps.gob.ec/documents/20181/564402/ Calificaci\%C3\%B3n+de+Riesgo + mar zo+2018.pdf/2ee31045-78aa-42c1-9730aa83da75db32

Marrón, E. (2015). La evitación al trabajo en las organizaciones santarroseñas y su relación con la cultura organizacional. Maestría en Gestión Empresaria, Administración de Recursos Humanos, Tesis. La Pampa, Argentina: Universidad Nacional de La Pampa.

Martínez, M. (2012). La cultura de empresa. En M. Martínez, La gestión empresarial. Madrid: Ediciones Díaz de Santos.

Miquilena, D. y Paz, A. (2008). La cultura como fuente de compromiso del capital humano 
en las organizaciones modernas. Forum Humanes, 1 (1), 1-12.

Mishkin, F. (2008). Moneda, banca y mercados financieros (8va ed.). México: Pearson Educación. PFEFFER, J. (2000). Nuevos rumbos en la teoría de la organización: problemas y posibilidades. México, D.F.: Oxford University Press.
Rodríguez, R. (2009). La cultura organizacional. Un potencial activo desde la perspectiva de la organización. Invenio, 12 (22), 67-92. Vargas, J. (2007). La culturocracia organizacional en México. Jalisco, México: Eumed.net. 